\title{
On the theory of relativistic collapse and relativistic explosion. 1. Collapse without horizon and formation of frozars.
}

\author{
Zahid Zakir $^{1}$
}

\begin{abstract}
In general relativity (GR) the surface of a spherical object as an extended body is defined on hypersurfaces of simultaneity $\mathrm{t}=\mathrm{const}$ and the proper time on the object's surface at any moment is strongly related to the finite world time moment $t$ (astronomical epoch). At any finite $t$ this proper time moment always is less than its value at which the surface would cross the gravitational radius in the Newtonian theory. It is shown that in GR, as the surface closely approaches the gravitational radius, the proper times rapidly freeze (in terms of $t$ ) firstly at the center, where the time delay is maximal, then at higher layers, and that the surface freezes after all layers beyond the gravitational radius. The freezing of all processes in entire volume means the practically stopping of the collapse also. Thus, in GR there is a universal factor preventing the collapse - a strong gravitational dilation of the proper times, a fundamental physical phenomenon with which GR mainly differs from the Newtonian theory. For this reason in GR the horizons and singularities, as the Newtonian theory artifacts, do not arise and the black holes are forbidden. The collapse in GR leads to the formation of the frozars (frozen stars) the surface and all layers in entire volume of which are frozen. The worldlines of the particles in the star are timelike and almost parallel to the $\mathrm{t}$-axis and between each other. The formation of the frozars is shown for the standard idealized models of the collapse - a thin dust shell, a dust ball, a star of constant density and a star with ultrarelativistic matter. The astrophysical consequences of the theory of collapsed stars as frozars of GR are shortly discussed.
\end{abstract}

PACS: 04.20.Dg; 04.70.-s; 97.60.-s, 98.54.-h

Key words: relativistic stars, collapse, frozars, black holes, horizon, singularity, time dilation

\section{Content}

Introduction 1

1. A gravitationally freezing dust shell as a simplest frozar ..................................................................... 3

2. The frozar formation at contraction of a homogeneous dust ball....................................................

3. The frozar formation at non-uniformly contracting dust ball .........................................................8

4. Frozar formation at collapse of homogeneous fluid and ultrarelativistic matter ...........................10

5. The structure and properties of compact objects in the frozar phase .............................................. 11

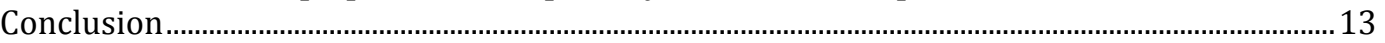

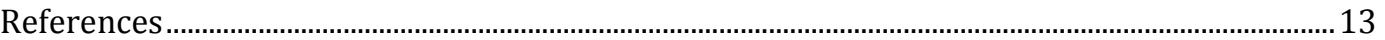

\section{Introduction}

The gravitational collapse long time remained one of main unsolved problems of astrophysics [1-9]. Only recently it became clear, how general relativity (GR) simply and fundamentally solves this problem [10].

${ }^{1}$ Centre for Theoretical Physics and Astrophyics, Tashkent, Uzbekistan; zahidzakir@ theor-phys.org 
At stellar collapse in the Newtonian physics, where time is absolute, the surface rapidly would cross the gravitational radius $r_{g}=2 G M$ and the star would become black since light could not escape it and a hole since all matter of the star would fall to the center by forming a physical singularity. Therefore, the possible formation of a black hole at the gravitational collapse is the prediction of the Newtonian physics and history of this fact is well-known.

During last more than half century as a theory of the collapse it has been accepted some hybrid of this Newtonian picture with GR in the form of the black hole hypothesis (BHH) [4-9]. But in GR the worldline of a particle on the surface does not cross $r_{g}$ for distant observers at any finite (astronomical) world time moment $t<\infty$. Nevertheless in $\mathrm{BHH}$ it has been declared that it easily crosses $r_{g}$ for local observers in terms of their proper time $\tau$. Such dual picture could be possible, obviously, only if it's proper time evolution along the worldline $r(\tau)$ could be occurred independently on its world time evolution $r(t)$. Thus, the black hole paradigm is based on an implicit hypothesis that with the same particle can occur two different sets of events for two groups of observers with qualitatively different behavior of the worldlines.

In fact, this intuitively introduced hypothesis not only makes the theory internally contradictory, moreover, it is mistaken in the framework of GR. In GR the worldline of the particle, as a unique set of events occurred by it, is unique and invariant. The proper time moment $\tau$ of the particle at $r$ and corresponding world time moment $t$ are only two parametrizations of the same event on the same world line $r(\tau) \equiv r(t)$. As a result, the equations of motion in GR give a one to one correspondence $\tau[r(t)] \equiv \tau(t)$ between the proper time moments $\tau$ and the world time moments $t$ on this unique world line. The proper time rate along the world line is strongly constrained by the world time rate and an event which still does not occur for the distant observers at a world time moment $t<\infty$, still does not occur for the local observers in terms of their proper times also.

Thus, the BH hypothesis, based on the mistaken in GR implicit assumptions, should be rejected as giving a wrong quasi-Newtonian picture of the gravitational collapse and forming at this process objects.

A way to the formulating an adequate to GR theory of the gravitational collapse has been found in 2006 [10]. As a physical basis it has been taken the fact that in GR there is a new fundamental factor for the stabilization of the collapse, absent in the Newtonian theory, and which has not been properly understood and taken into account till now. It is the gravitational slowering of the proper times with respect to the world time because of which the worldline of the surface never crosses $r_{g}$ at any finite world time moment $t<\infty$ for all observers with any coordinates. In the extremely strong gravitational field of the collapsing star it leads to the practically freezing of all processes in entire volume of the star with respect to the (astronomical) world time by forming of $a$ frozar ("frozen star").

The qualitatively new properties of the relativistic collapse there demonstrate the standard exactly solvable models of the stellar collapse. In the previous papers [10] it has been considered the freezing of a contracting thin dust shell by forming a simplest frozar. It has been shown that shell's radius $r_{b}$ asymptotically approaches $r_{g}$ and, due to the 
invariance of the worldline, the shell locates outside it $\left(r_{b}(t)>r_{g}\right)$ at any $t<\infty$ for all observers. Main results of this analysis are presented in Section 1 of the present paper.

In other sections of the present paper the properties of the relativistic collapse and the structure of the forming frozars are studied firstly on the example of a dust ball and then for the models with non-zero pressure - a star of constant density and a star with ultrarelativistic matter. Astrophysical consequences of the theory of compact relativistic stars as frozars are discussed also.

\section{A gravitationally freezing dust shell as a simplest frozar}

General properties of compact relativistic objects in GR can be revealed in the case of a simplest and exactly solvable model of a thin dust shell freely falling in own gravitational field. In this case the dust particles are at the same time both a source of the field and the test particles, so here two problems - the definition of structure of the source and motion of test particles - are solved simultaneously. In the previous papers [10] the theory of compact objects as frozars has been developed on the basis of this model.

The gravitational field of a spherical non-rotating object of radius $r_{b}$ in the rest frame of its center is centrally symmetric and static in the region $r \geq r_{b}$. As it is well known, in the static field it is possible to define the simultaneity of distant events, which allows synchronizing clocks in entire region $r \geq r_{b}$ at any moment. As the result, there are the hypersurfaces of simultaneity $t=$ const, where the synchronized coordinate clocks show the global world time $t$ of simultaneously occurring events. The source as an extended object is defined on such hypersurface as "a matter located in the region $r \leq r_{b}\left(t_{0}\right)$ at a moment $t_{0} "$.

Notice, that the rest frame of the source with the curvature coordinates $(t, r, \theta, \varphi)$ is physically preferred in that sense that: (1) there is a global simultaneity of events labeled by the world time $t$, (2) the world time intervals are equidistant everywhere in the field and at any time, (3) the radial velocities of shell's particles under the center are the same and (4) the shell is spherically symmetric. In any other frame some of these four properties disappear.

In the curvature coordinates the metrics on and outside the shell $\left(r \geq r_{b}\right)$ are given by the Schwarzschild solution:

$$
d s^{2}=e^{v_{s}(r)} d t^{2}-e^{\lambda_{s}(r)} d r^{2}-r^{2}\left(d \theta^{2}+\sin ^{2} \theta d \varphi^{2}\right), \quad r \geq r_{b},
$$

where $\exp \left[v_{s}(r)\right]=\exp \left[-\lambda_{s}(r)\right]=1-r_{g} / r, r_{g}=2 G M$. At $r<r_{b}$ the internal metrics are regular, the spatial geometry is flat and at any point the slowing down of proper times is as on the surface.

Let a dust shell starts to falling from some large radius $r_{b}\left(t_{0}\right) \gg r_{g}$ at $t_{0}=0$ and $\tau_{0}=0$. There world lines of the dust shell's particles will place outside the gravitational radius at any finite world time moment $t>0$, with which the radial coordinate $r(t)$ is related as:

$$
t=C\left(r_{1}\right)-2\left(r r_{g}\right)^{1 / 2}-\frac{2}{3} \frac{r^{3 / 2}}{r_{g}^{1 / 2}}+r_{g} \ln \left|\frac{r^{1 / 2}+r_{g}^{1 / 2}}{r^{1 / 2}-r_{g}^{1 / 2}}\right|,
$$


where a constant $C\left(r_{1}\right)=$ const is chosen so that it should be $t\left(r_{1}\right)=0$. For large enough times $r_{g} \ll t<\infty$ the formula simplifies and an explicit relation is very simple:

$$
r(t)=r_{g}\left(1+4 \mathrm{e}^{-t / r_{g}}\right)+O\left(r_{g} / t\right)>r_{g} .
$$

At the parametrization of the same world line by the proper time a formula for the trajectory has the form:

$$
r(\tau)=r_{0} \cdot\left(1-\tau / \alpha_{0}\right)^{2 / 3},
$$

where $\alpha_{0}=2 r_{0}^{3 / 2} / 3 r_{g}^{1 / 2}$, and is the same as in the Newtonian theory. The proper time moment $\tau_{g}$ at which the shell would cross $r_{g}$ in the Newtonian theory, follows from (4):

$$
\tau_{g}=\alpha_{0} \cdot\left(1-\frac{r_{g}^{3 / 2}}{r_{0}^{3 / 2}}\right)
$$

Two formulas for the trajectory (3) and (4) describe the same particle at the same spatial point $(r, \theta, \varphi)$ in terms of indications of two kind of clocks. Therefore, in fact there is an identity $r(\tau) \equiv r(t)$, in (3) and (4) at $r_{g} \ll t<\infty$ and there is a one-to-one correspondence between the proper time moments $\tau[r(t)] \equiv \tau(t)$ and the world time moments $t$ (Fig. 1) [7]:

$$
\tau(t)=\alpha_{0}\left[1-\frac{r_{g}^{3 / 2}}{r_{0}^{3 / 2}}\left(1+4 \mathrm{e}^{-t / r_{g}}\right)^{3 / 2}\right]<\tau_{g} .
$$

Here $\tau(t)<\tau_{g}$ at any $t<\infty$ and the moment $\tau_{g}$ will not come. This means, that at starting the falling of the surface from an initial value $\tau\left(r_{0}\right)=0$ at $t_{0}=0$, the infinite world time interval $t \in[0, \infty)$ will be mapped on a finite interval of its proper time $\tau(t) \in\left[0, \tau_{g}\right)$. Thus, the effect of the gravitational time dilation reveals in the form of the actual freezing of the proper times at close approaching to $r_{g}$. Thus, in GR the rate of the proper times is strongly restricted by the uniform rate of the world time on the global hypersurfaces of simultaneity $t=$ const (Fig. 1).

Because of the invariance of the proper times the dust ball never crosses the gravitational radius $\tau\left[t\left(t^{\prime}, r^{\prime}\right)\right]>\tau_{g}$ for any observers with any coordinates $t^{\prime}, r^{\prime}$, corresponding to a finite astronomical world time moment $t<\infty$ of the real world. The observers in any frame will see the same picture at comparing their coordinates with the really occurring events on the hypersurfaces of simultaneity of the dust ball's rest frame.

In the internal area of the dust shell $r<r_{b}$ where the time delay factor is equal to the value on the surface, the event horizon also does not appear, but all test particles will be frozen in those points where they have appeared up to a shell's freezing time near $r_{g}$. Therefore, for the (massive) test particles in the shell's gravitational field the spacetime interval remains timelike $d s^{2}>0$ at any spatial point $r \geq 0$.

Let's consider the trajectories of the particles of the contracted shell on the plane $(t, r)$ showed in the Fig. 2. In this diagram the world time intervals along the time axis are equidistant since $t$ is defined globally. Since the proper times $\tau(r)$ are defined locally at the vicinity of each world line, their intervals are not equidistant and, 
consequently, the moments $\tau(r)$ should be arranged only along each world line. As the trajectories asymptotically approaches $r_{g}$ at the uniformly going world time, the proper time intervals become more and more stretched and the value $\tau(r)$ practically stops (frosts) near $\tau_{g}$. The freezing of the shell is appeared as an apparent "breaking" of the worldline $r(\tau)$ near $r_{g}$, while in fact the evolution is continued in the form of microscopic shifts $r \rightarrow r_{g}$, which are shown more clearly in the same worldline $r(t)$ represented in terms $t$.

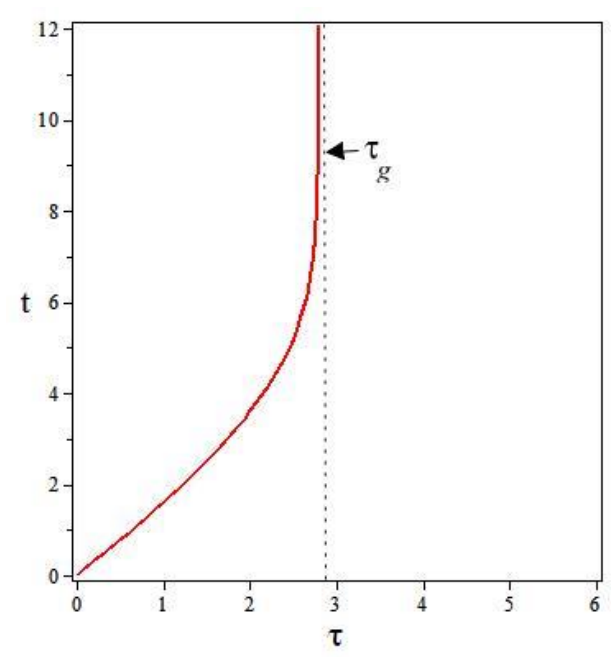

Fig. 1. The proper time $\tau=\tau[r(t)]=\tau(t)$ of a dust shell is restricted due to the relation $\tau(t)=\tau_{g}-b \exp \left(-t / r_{g}\right)<\tau_{g}$ at any $t<\infty$.

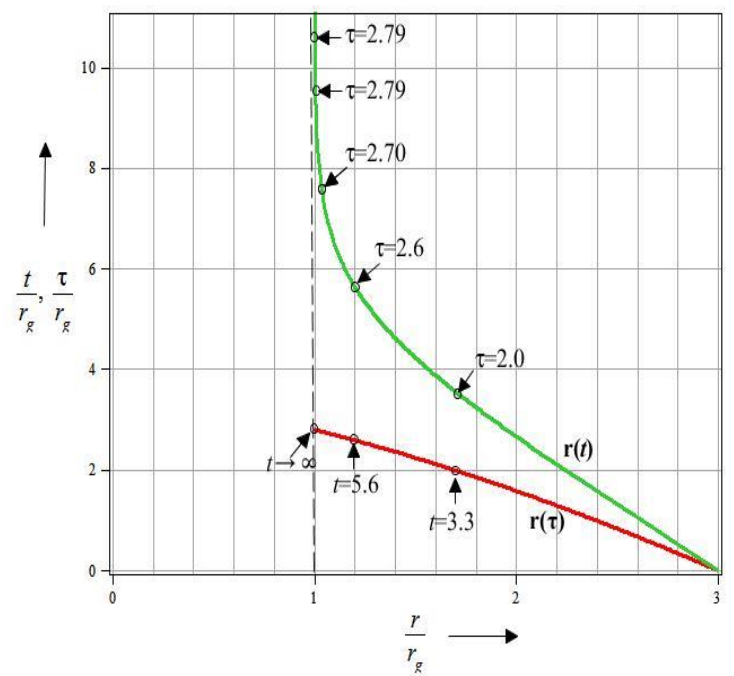

Fig. 2. An invariant worldline of a contracting dust shell $r(t)=r(\tau)$ represented in terms of world time $t$ and proper time $\tau$.

A conclusion analogous to the dust shell holds in the cases of a thin shell of weakly charged particles or a rotating (neutral or charged) shell falling in own field. There are two forms of the equation of trajectory in terms of the world time or the proper times of the falling particles. These equations of the trajectory again will give the equations relating the proper times $\tau(t)$ with the world time moments $t<\infty$, the particles on the surface also remain outside an area where the proper times practically freeze. In the case of the charged shell - outside an effective gravitational radius $\tilde{r}_{g}(r)=r_{g}-G e^{2} / r$, and in the case of the neutral rotating shell - outside the ergosphere, where $g_{00}(r, \theta) \rightarrow 0$ and the proper times freeze with respect to the world time.

\section{The frozar formation at contraction of a homogeneous dust ball}

A homogeneous dust ball can be considered as a system of enclosed thin dust shells, where each layer of a finite mass changes the proper time dilation factor in any point of the volume to a finite value. The freezing of the surface in world time occurs according to the Schwarzschild solution and, therefore, it happens exponentially fast. However, since the time dilation on the internal layers is not less than on the surface, then the faster freezing of the proper times in the internal layers occurs before the freezing of 
the surface [10]. Below this essentially important property of the relativistic collapse will be shown in the cases of the standard exact solutions of the field equations.

For the model of a contracting dust ball a general solution has been found by Tolman [5] (notations see [9]) with two unknown functions for a layer $R: f(R)$ proportional to the layer's initial energy and $F(R)$ - proportional to the mass inside the layer. Let us consider the case $f(R)<0$ (elliptic trajectories) for a standard problem of a homogeneous dust ball, the particles of which has rested $\dot{r}\left(R, t_{0}\right)=0$ at initial moment $t_{0}=0$ (see [9], a problem to $\S 103$ ). Let us show in this case the formation of the frozen ball as transition to the frozar state.

The solution is given parametrically by the equation for the cycloid. Let at the initial proper time moment $\tau_{0}(R)=0$ the cycloid's parameter is chosen as $\eta_{0}=0$ and the perimeter of ball's surface is equal to $2 \pi r_{0}$. When the functions $f(R)$ and $F(R)$ are chosen in the form:

$$
f=-\sin ^{2} R, \quad F=2 a_{0} \sin ^{3} R,
$$

this corresponds to the isotropic Friedmann solution with the scale factor $a(\eta)$, where the parameter $\eta(t)$ is related to $\tau$ as $d \tau=a d \eta$, while the line element is:

$$
d s^{2}=a(\eta)\left[d \eta^{2}-d R^{2}-\sin ^{2} R\left(d \theta^{2}+\sin ^{2} \theta d \varphi^{2}\right)\right] .
$$

The initial value of the scale factor can be fixed by the matching with the external metrics of the ball, which gives $a_{0}=r_{0} / 2 \sin R_{0}$, where $r_{g}=r_{0} \sin ^{2} R_{0}$ is the gravitational radius of the ball, and $R_{0}$ labels the surface. Thus, the isotropy and uniformity of the ball appears in the fact that the proper time values $\tau(t)$ at each moment $t$ are the same on all layers, do not depend on $R$ and are defined by the "epoch" $t$ only:

$$
\tau(t)=\frac{r_{0}^{3 / 2}}{2 r_{g}^{1 / 2}}[\eta(t)+\sin \eta(t)], \quad r(t, R)=\frac{r_{0}^{3 / 2}}{2 r_{g}^{1 / 2}}[1+\cos \eta(t)] \sin R,
$$

The matching with the external metrics allows us to relate $\eta$ to $t$ :

$$
\frac{t}{r_{g}}=\ln \frac{\left(r_{0} / r_{g}-1\right)^{1 / 2}+\tan (\eta / 2)}{\left(r_{0} / r_{g}-1\right)^{1 / 2}-\tan (\eta / 2)}+\left(r_{0} / r_{g}-1\right)^{1 / 2}\left[\eta+\frac{r_{0}}{2 r_{g}}(\eta+\sin \eta)\right] .
$$

It is clear from this formula that $\eta(t)$ is a function of the time $t$ only.

At once it is essentially important to what a limit tends the proper time $\tau(t)$ at $t \rightarrow \infty$. As shows (10), at $t \rightarrow \infty$ we have $\tan (\eta / 2) \rightarrow\left(r_{0} / r_{g}-1\right)^{1 / 2}$ and $\eta$ tends to a finite limit:

$$
\left.\eta(t)\right|_{t \rightarrow \infty} \rightarrow \eta_{g}=2 \arccos \left[\left(r_{g} / r_{0}\right)^{1 / 2}\right] .
$$

It means that in this case the infinite world time interval $t \in[0, \infty)$ is mapped on a finite interval of this parameter $\eta(t) \in\left[0, \eta_{g}\right)$. As the ball contracts and its surface closely approaches $r_{g}$, there occurs the exponentially fast freezing of the surface under the law (9). The internal layers freeze under the same law, since, due to homogeneity and 
isotropy, the proper times everywhere are the same. For the value $\tau_{g}$, at which the proper times asymptotically freeze, then we find:

$$
\begin{gathered}
\left.\tau(t)\right|_{t \rightarrow \infty} \rightarrow \frac{r_{0}^{3 / 2}}{2 r_{g}^{1 / 2}}\left(\eta_{g}+\sin \eta_{g}\right) \equiv \tau_{g}, \\
\tau_{g}=\frac{r_{0}^{3 / 2}}{r_{g}^{1 / 2}} \arccos \left[\left(r_{g} / r_{0}\right)^{1 / 2}\right]+r_{0} \cdot\left(1-r_{g} / r_{0}\right)^{1 / 2} .
\end{gathered}
$$

Thus, the moments from the infinite interval $t \in[0, \infty)$ one to one correspond to the moments from the finite proper time interval $\tau(t) \in\left[0, \tau_{g}\right)$, which expresses a practically stopping the proper times in entire ball at $t \gg r_{g}$.

Ball's internal layers, thus, freeze at the values of the radial coordinate:

$$
r(\infty, R)=\frac{r_{0}^{3 / 2}}{2 r_{g}^{1 / 2}}\left(1+\cos \eta_{g}\right) \sin R=\left(r_{0} r_{g}\right)^{1 / 2} \sin R
$$

As we see, the surface freezes nearby $r\left(\infty, R_{0}\right) \rightarrow r_{g}$, and other layers will be distributed sinusoidal on certain distances from the ball's center. Further the world lines of ball's particles with a high accuracy remain parallel to the axis $t$ and to each other.
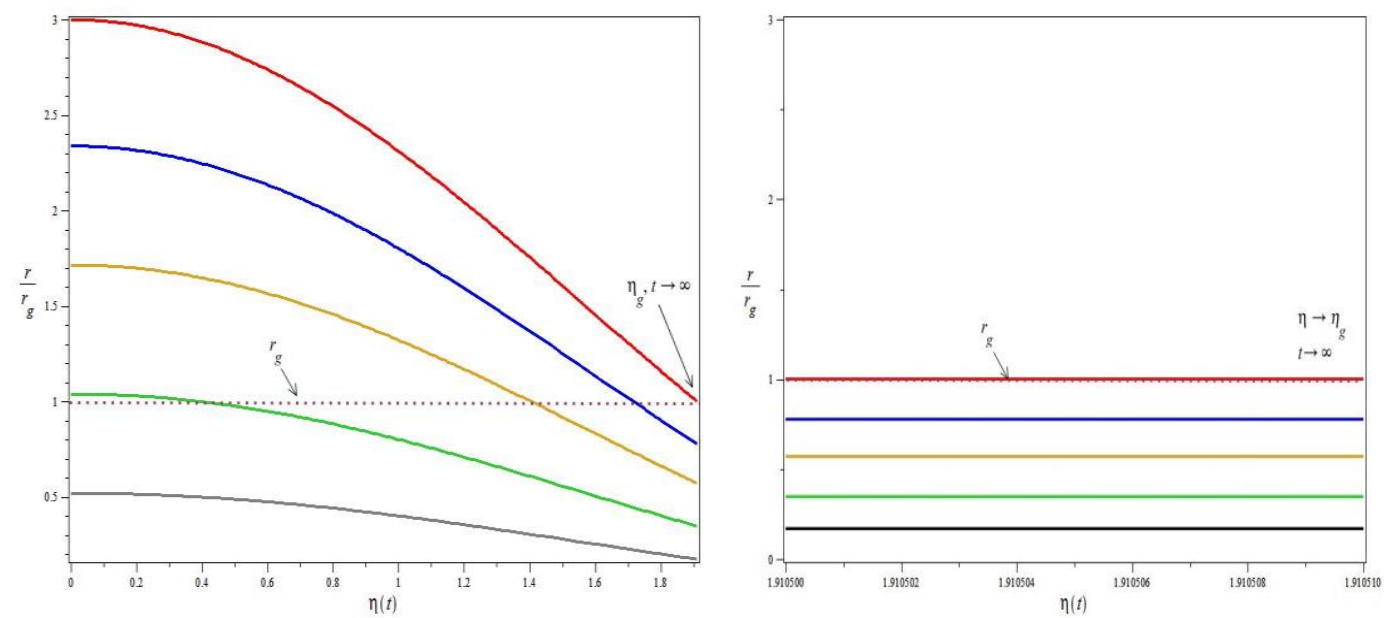

Fig. 3. Decreasing of radial coordinates of layers in a dust ball in world time $t$ expressed through the parameter $\eta(t)$. Its surface will promptly come near a value of the parameter $\eta \rightarrow \eta_{g}=2 \arctan (\sqrt{2}) \approx 1.910633236$, where this parameter, proper times and length of perimeters of layers freeze in world time and further do not change up to $t \rightarrow \infty$. The surface freezes outside the gravitational radius and after the freezing the ball remains homogeneous.

For the scale factor of the Friedmann metrics in this model and its limiting value $a_{g}$ at which the ball freezes, we obtain:

$$
a(t)=\frac{r_{0}^{3 / 2}}{2 r_{g}^{1 / 2}}[1+\cos \eta(t)], \quad a_{g}=\frac{r_{0}^{3 / 2}}{2 r_{g}^{1 / 2}}\left(1+\cos \eta_{g}\right)=\left(r_{0} r_{g}\right)^{1 / 2} .
$$

At the initial density $\rho_{0}$ the corresponding limiting density is equal to: 


$$
\rho_{g}=\rho_{0} \frac{a_{0}^{3}}{a_{g}^{3}}=\rho_{0} \frac{1}{\left(r_{0} r_{g}\right)^{3 / 2}} \frac{r_{0}^{9 / 2}}{r_{g}^{3 / 2}}=\rho_{0} \frac{r_{0}^{3}}{r_{g}^{3}} .
$$

Thus, the homogeneous dust ball with the standard idealizations and the initial conditions, leading to the Friedmann metrics, freezes very quickly at the proper time value $\tau(t) \rightarrow \tau_{g}$, the radial coordinates of the layers $r(t, R) \rightarrow\left(r_{0} r_{g}\right)^{1 / 2} \sin R$ and the density $\rho(t) \rightarrow \rho_{0} r_{0}^{3} / r_{g}^{3}$, so that at $t \rightarrow \infty$ already there never happens with this ball. Thus, the dust ball passes into the frozar state - a star with completely frozen internal structure which remains almost unchanged at its coexistence with other objects of the Universe at any $t<\infty$.

\section{The frozar formation at non-uniformly contracting dust ball}

For a dust ball considered in the previous section the isotropic Friedmann solution is applicable only approximately since in fact at the central symmetry the acceleration directed to the center is non-zero and isotropy is broken by it [3-4]. It appears, particularly, in the dependence on the radial coordinate of the time component of the metrics and the proper time in the ball is slowed down non-uniformly.

In a more rigorous approach to the dust ball there appear two new properties with respect to a thin dust shell, characteristic for other compact relativistic objects also.

The first property is that a spatial contraction factor $\exp [\lambda(t, r)]$, which on the surface is equal to the Schwarzschild's value, is non-uniform and decreases up to unity at the center: $\exp [\lambda(t, 0)] \rightarrow 1$.

The second new property is the increasing of the time delay factor $\exp [-v(t, r)]$ at approaching the ball's center. Since on the surface it also is equal to the Schwarzschild value, this means that, as the surface closely approaches $r_{g}$, the central parts of the ball freeze faster than the higher layers at smaller values of $\tau$ then at the surface. As the result, on each hypersurface of simultaneity $t=$ const the following inequality takes place (at starting from the zero initial values $\tau(r, 0)=\tau\left(r_{b}, 0\right)=0$ ):

$$
\tau(r, t) \leq \tau\left(r_{b}, t\right), \quad r \leq r_{b} .
$$

All these are evident on the well-known solution of the Einstein equations given by Oppenheimer and Snyder (OS) [4]. At $t \gg r_{g}$ the dependence between two times $\tau(t)$ in this solution in the reversed form is (with a corrected coefficient according [5]):

$$
t \approx-r_{g} \ln \left[\frac{1}{8}\left(\frac{R^{2}}{R_{b}^{2}}-3\right)+\frac{R_{b}}{4 r_{g}}\left(1-\tau / \alpha_{b}\right)^{2 / 3}\right]+O\left(r_{g} / t\right),
$$

where $\alpha_{b}=2 R_{b}^{3 / 2} / 3 r_{g}^{1 / 2}$, and $R$ is found from the expression:

$$
r(t)=R \cdot\left[1-\tau(t) / \alpha_{b}\right]^{2 / 3},
$$

$R_{b}$ is a value of this variable on the ball's surface and $R=0$ at the center.

For understanding of a physical meaning of the dependence (18) it is more useful a direct form $\tau(t, R)$ where the proper time of each layer is expressed through $t$ : 


$$
\tau(t, R)=\alpha_{b}\left\{1-\frac{r_{g}^{3 / 2}}{R_{b}^{3 / 2}}\left[4 e^{-t / r_{g}}+\frac{1}{2}\left(3-\frac{R^{2}}{R_{b}^{2}}\right)\right]^{3 / 2}\right\} .
$$

At $t \gg r_{g}$ the proper times of layers exponentially quickly freeze about maximal values:

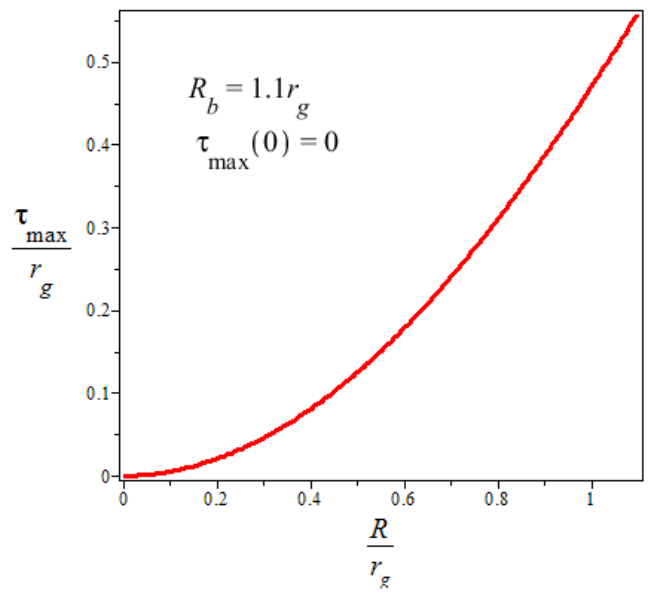

Fig. 4. Inhomogeneous freezing of proper times of dust shell's layers beginning from a freezing moment of the center $\tau_{\max }(0)$.

$$
\tau_{\max }=\alpha_{b}\left\{1-\left[\frac{r_{g}}{2 R_{b}}\left(3-\frac{R^{2}}{R_{b}^{2}}\right)\right]^{3 / 2}\right\}
$$

The diagram of the dependence $\tau_{\max }(R)$ is shown in a Fig. 4. As we see, the proper time at the surface $R=R_{b}$ freezes at a value:

$$
\tau\left(t, R_{b}\right) \rightarrow \alpha_{b}\left(1-r_{g}^{3 / 2} / R_{b}^{3 / 2}\right) .
$$

Since at the center of the ball $R=0$ the proper time delay is stronger, it asymptotically freezes earlier the surface at a value $\tau_{\text {max }}(0)<\tau_{\max }\left(R_{b}\right)$ :

$$
\tau(t, 0) \rightarrow \alpha_{b}\left[1-\left(3 r_{g} / 2 R_{b}\right)^{3 / 2}\right] \text {. }
$$

A difference in the freezing proper times on the surface and at the center (in terms $t$ ) remains finite:

$$
\Delta \tau_{\infty}=\tau\left(\infty, R_{b}\right)-\tau(\infty, 0)=\left(\sqrt{\frac{3}{2}}-\frac{2}{3}\right) r_{g} \approx 0,558 r_{g} .
$$

Thus, at any distribution of density in the ball the time delay factor will be maximal at the center and minimal on the surface and the center freezes faster than the surface (Fig. 4).

Notice, that if we do not neglect pressure the time dilation coefficient formally diverges already at finite distance from the center. However, in real stars this will not happen since the rapid increasing of pressure and temperature at the center will lead to the changing of an equation of state and the initial model assumptions should be improved.

The radial contraction factor in the OS solution looks like (with a corrected coefficient as in [5]):

$$
e^{-\lambda(r, t)}=1-\frac{R^{2}}{R_{b}^{2}}\left[4 e^{-t(r) / r_{g}}+\frac{1}{2}\left(3-\frac{R^{2}}{R_{b}^{2}}\right)\right]^{-1}
$$

At the center $R=0$ we have $\lambda(0, t)=0$ at any moment. In the higher layers the radial contraction factor grows and as the surface closely approaches to $r_{g}$, the physical volume quickly increases because of the radial contraction in the higher layers. Thus, the radial contraction factor in the higher layers increases in world time exponentially:

$$
e^{\lambda(r, t)} \sim e^{t(r) / r_{g}}+\frac{\varepsilon}{R_{b}} \rightarrow e^{t(r) / r_{g}} .
$$

Separate layers of the dust ball, with different $R$ at the beginning, quickly freeze (i.e. do not depend from $t$ ) at the values of the radial coordinate: 


$$
r=\frac{R}{R_{b}}\left[\frac{r_{g}}{2 R_{b}^{1 / 3}}\left(3-\frac{R^{2}}{R_{b}^{2}}\right)\right]^{3 / 2} .
$$

The third new property is connected with removal for the dust ball of known restrictions on the surface radius and on the redshift $z$, arising for objects in hydrostatic equilibrium because of unlimited increase of pressure at the center (see [8]):

$$
r_{b}>\frac{9}{8} r_{g}, \quad z<2
$$

Really, in the contracting dust ball there is no pressure and it is not in equilibrium, so formally this restriction does not concern it.

At last, the fourth property distinguishing a relativistic dust ball from the black holes of the Newtonian theory is that all trajectories of particles are timelike $d s^{2}>0$ in entire volume, the surface remains always beyond the gravitational radius $r_{b}>r_{g}$ and, consequently, the particles and radiation in principle can leave the dust ball (at the account of temperature effects, through a large time and with a very large redshift).

Thus, in process of ball's contraction together with increase of physical volume and number of particles in the higher layers, the time delay effect in the lower layers will increases. The freezing of all processes, having begun at the center, will extend on the higher layers also, so the contracted dust ball in terms of the world time quickly freezes at a surface radius very close to $r_{g}$ and further its structure on entire volume remains unchanged, i.e. OS solution leads to the formation of the frozar.

\section{Frozar formation at collapse of homogeneous fluid and ultrarelativistic matter}

The process of formation of frozars at the collapse at zero pressure (a thin dust sphere and a dust ball), considered in above two sections, has a general character and conclusions about the collapse at non-zero pressure will be qualitatively the same at a soft equation of state. Further it will be shown for two standard exactly solvable stellar models with non-zero pressure.

Let us consider a static homogeneous star having mass close to a critical mass, when at adiabatically increasing of the mass $r_{g}$ grows and becomes close to the surface radius $r_{b}$. As a rule, there is another critical value $r_{c}$ (for example, $9 r_{g} / 8$ ) after which the stability will be lost, but it also is close enough to $r_{g}$. Let the matter density in a cold homogeneous star is constant: $\rho(r)=\rho_{0}=$ const. In this case the time component of the metric $g_{00}(r)$ and the pressure $p(r)$ are expressed through $r_{b}$ and the total mass $M=4 \pi \rho_{0} / 3 r_{b}^{3}$ in the standard form [8]:

$$
\begin{gathered}
g_{00}(r)=\frac{1}{4}\left[3\left(1-r_{g} / r_{b}\right)^{1 / 2}-\left(1-r_{g} r^{2} / r_{b}^{3}\right)^{1 / 2}\right]^{2}, \\
p(r)=\frac{3 M}{8 \pi r_{b}^{3} g_{00}^{1 / 2}(r)} \cdot\left[\left(1-r_{g} r^{2} / r_{b}^{3}\right)^{1 / 2}-\left(1-r_{g} / r_{b}\right)^{1 / 2}\right] .
\end{gathered}
$$

Here the time dilation factor $g_{00}^{-1 / 2}(r)$ also is minimal on the surface and maximal at the center: 


$$
\begin{gathered}
g_{00}^{-1 / 2}\left(r_{b}\right)=2\left[3\left(1-r_{g} / r_{b}\right)^{1 / 2}-\left(1-r_{g} / r_{b}\right)^{1 / 2}\right]^{-1}, \\
g_{00}^{-1 / 2}(0)=2\left[3\left(1-r_{g} / r_{b}\right)^{1 / 2}-1\right]^{-1}>g_{00}^{-1 / 2}\left(r_{b}\right) .
\end{gathered}
$$

As a result, even if the star loses stability and its surface becomes very close to $r_{g}$, where the proper time starts to freeze exponentially quickly, the proper times of particles near the center and in other internal layers freeze before and more quickly than at the surface. Thus, while we neglect a possible scenarios of the stabilization or anticollapse and consider a worst ("pessimistic") scenario when the contraction is inevitable, the including of the pressure reveals only on a dependence of the time dilation factor on the layers, but it does not change a character of the freezing of the star as a whole with formation of a frozar.

Let's consider another model of a relativistic star, the main part of matter in which is the ultrarelativistic particles with the equation of state $p(r)=\rho(r) / 3$. In Such star the temperature effects are essential everywhere due to $\rho(r)=\sigma T^{4}(r)$. However, as an abstract model we continue to consider star's dynamics as for a cold case.

Corresponding time component of the metrics on the intermediate layers of such star has a very simple form [4]:

$$
g_{00}(r)=\frac{r}{r_{b}}\left(1-\frac{2 G M\left(r_{b}\right)}{r_{b}}\right)=\frac{4}{7} \frac{r}{r_{b}}, \quad r \leq r_{b} .
$$

As the result, the time dilation factor, which is finite on the surface, rapidly increases near the center:

$$
g_{00}^{-1 / 2}\left(r_{b}\right)=\sqrt{7} / 2, \quad g_{00}^{-1 / 2}(0) \rightarrow \infty
$$

Thus, at the central regions of such star the proper times of particles already are frozen even long before the star's collapse. In fact, the center of the star with ultrarelativistic matter always is in a frozar state (again in the "pessimistic" scenario when the pressure and temperature do not prevent the contraction). At loss of stability and further contraction of the star with transition into a quick collapse mode, this already existing internal frozar near the central region will extend by including higher layers as surface approaches close to $r_{g}$, after which the star already completely turns into the frozar state.

\section{The structure and properties of compact objects in the frozar phase}

In more realistic models of relativistic stars, in which both the pressure and temperature are taken into account, the picture of the asymptotical contraction with freezing will be replaced to a more non-trivial behavior of layers. Nevertheless, there maintain basic properties of relativistic collapse shown for the simpler cases, in addition qualitatively new properties will appear also.

From the former properties the main is that all relativistic objects appear as stable under the gravitational contraction due to almost a total gravitational freezing of the star (in world time) as its surface approaches $r_{g}$ and internal regions freeze before a surface.

New properties which were absent in the dust matter, consist in that at certain structure and an equation of state, the gravitational contraction of the star can transfer into a quasi-stable state or into the expansion with observable radiations and ejections.

At the contraction of the star the physical volume in higher layers increases rather 
faster, than in the bottom layers. Firstly, in the higher layers the radial contractions are stronger, and secondly the higher layers of the star at any equation of state freeze after the central ones and have time to be condensed longer and up to higher values. Thus, initially homogeneous star at the contraction becomes inhomogeneous and for such stars there will be no restrictions for the redshifts of the radiation from the surface, as for the equilibrium state stars.

At the close approaching $r_{g}$ by the surface, the local pressure and temperature near the center rapidly increases. This can lead to the compensation of the contraction, open new channels of reactions by transformations of particles and also lead to the phase transitions. Energy releasing at such reactions and phase transitions can lead to star's stabilization on any equilibrium value of parameters or to the expansion. Particularly, at first the central, and then the higher layers can transform into the quark phase with the energy releasing, allowing stabilizing contraction or leading to large emissions and star's explosion.

Let's discuss some other general properties of the compact relativistic stars (frozars) following from the GR restrictions and showed in the simple models:

1. The large time delay near the surface of the frozars leads to the absence of the sharp changes of parameters and to a very smooth and stretched in time character of all peaks of intensity of radiation at accretion. Such slowing down can be used for the measuring of time delay factor near the frozars.

2. The silence at the final stage of matter's falling to the frozars due to the gravitational freezing of such matter and its radiation.

3. The gravitationally-frozen matter will be superdense only at the central regions of the supermassive frozars in quasars, galaxy nuclei and stellar clusters. In closer to the surface areas the matter's density may be low, although it will be also frozen in terms of $t$. Thus, the supermassive frozars will have a complex internal structure with matter in various states.

4. Quantum processes in a static field will not lead to a particle creation since all world lines are timelike and there is no direct quantum evaporation of the static frozar.

5. At vacuum fluctuations of quantum fields around the frozar one of quanta of a pair falls on the frozar, and another leaves its vicinity in terms of world time and if this is allowed by conservation laws (for example, at the presence of a thermostat or external fields), the leaving quanta will be in a mixed state describing by the density matrix. Such partial loss information because of the freezing a part of quantum fluctuations in the frozar would lead to appearance of effective entropy and a temperature. At such particle creation frozar's mass increases due to the absorption of a positive energy of one of particles of the created pair.

Some "candidates to collapsed objects" are more "silent", than neutron stars (or quark stars) and the thermonuclear flashes from the falling of matter on their surface do not observed. Some compact supermassive objects, such as galaxy nuclei also are "silent". These facts can confirm a new picture of the relativistic stars where the "flashes" and other locally-fast phenomena become strongly slowed down in terms of $t$. Such "slowed flashes", lasting hours and days are really observed practically for most part of such "silent" objects. Though the mechanisms of these phenomena may be complicated, nevertheless, the gravitational slowering of flashes, possibly, contribute to the total effect. 


\section{Conclusion}

As shows some simplest models of the relativistic collapse, as close the surface of a collapsing object to its gravitational radius $r_{g}$, as stronger becomes the gravitational freezing of proper times in entire volume of the object with respect to the astronomical world time $t$ of the Universe.

At a soft equation of state, when gravity dominates, at first freezes a center, then the freezing spreads to higher layers and a surface freezes after all near $r_{g}$. Thus, a proper time moment when the surface freezes, is slightly less than $\tau_{g}$ from (5). Such object with almost total frozen internal structure, where worldlines of particles in entire volume are timelike and are almost parallel to the $t$ axis and among themselves, is the frozar.

The frozars at any finite world time moment $t<\infty$ have a surface outside $r_{b}>r_{g}$ and inhomogeneous internal structure defined by the equation of state at the moment of freezing and a growing to surface radial contraction.

In contrast to the black holes with a horizon and singularity, which in fact are forbidden by GR, the frozars are observable in principle at any world time moment and can emit radiation with very large redshift. The observable types of the compact relativistic astrophysical objects (from stellar masses up to nuclei of galaxies, quasars and stellar clusters) can be interpreted in GR as the frozars of various mass, internal structure and nontrivial environment.

In the second part of the paper an "optimistic" scenario of the relativistic collapse will be considered, when "hard" equations of state at some combination of particles and fields stops or even reverse the contraction at some stage after enough strong freezing.

\section{References}

1. Schwarzschild K. (1916) Sitz. Preuss. Akad. Wiss., p. 189.

2. Lemaître (1933) Ann. Soc. Sci. Braxelles, Ser. A, 1933, v. 53, p.

3. Tolman (1934) Proc. Nat. Acad. Sci. US 20, 169.

4. Oppenheimer J.R., Snyder H. (1939) Phys. Rev. 56, p. 455.

5. Mitra A. (1999) arxiv:astro-ph/9904163.

6. Misner C.W., Thorne K.S., Wheeler J.A. Gravitation. Freeman, 1973.

7. Landau L.D., Lifshitz E.M. The Classical Theory of Fields. B.H. 1994.

8. Weinberg S. Gravitation and Cosmology. Wiley, 1972.

9. Frolov V.P., Novikov I.D. Black Hole Physics. Kluwer, 1998.

10. Zakir Z. Theor. Phys., Astrophys. and Cosmol., (2006) 1, 3, 42; (2007), 2, 1, 1; arXiv:0705.2585. 